\title{
Chinese Color Preference in Software Design
}

\author{
Yan Ge ${ }^{1,2}$, Ronggang Zhou ${ }^{1}, \mathrm{Xi} \mathrm{Liu}^{1}$, and Kan Zhang ${ }^{1}$ \\ ${ }^{1}$ State Key Lab of Brain and Cognitive Science, Institute of Psychology, \\ Chinese Academic of Sciences, \\ Beijing, 100101 \\ ${ }^{2}$ Graduate University of Chinese Academy of Sciences, \\ Beijing, China, 100049 \\ \{gey, zhangk\}@psych.ac.cn
}

\begin{abstract}
Three experiments were designed to investigate the color preference of User Interface of Chinese youth. Background color and foreground color, as well as their combinations were examined. The results showed that: 1, Blue, purple, gray-blue and cyan were the more popular background color. 2, the foreground color preference was influenced by the background color, but white, yellow series and green series were all popular foreground colors for the background color of blue, purple and gray-blue. The discrimination of participants for graphic was better than that for characters. Some mechanism and implications were discussed.
\end{abstract}

Keywords: Color preference; User interface; Background color; Foreground color.

\section{Introduction}

Psychologists have been taking great interesting on color preference for a long time, there were some studies on color preferences using printed cards in the past. Zhou \& Zeng (1986) ${ }^{[1]}$, Wang (1997) ${ }^{[2]}$ investigated color preference of Chinese students in different years. They found there was some difference in different years, but something was stable, e.g. white, green and blue were popular colors for Chinese students all the time. Li (1990) ${ }^{[3]}$ found color preferences were different for different objects; gender and age also influenced preference. Elli (2001) ${ }^{[4]}$ found significant gender difference in color preferences of North American college students. Today, the computer becomes more and more popular in the society, the study on color of computer user interface also attracts the attention of psychologists, but most of the studies mainly focused on the visual performance (Zhu \& Cao ${ }^{[5]}, 1994$; Cao \& Zhu ${ }^{[6]}$, 1995; Kong et al. $\left.{ }^{[7]}, 1999\right)$. No research was focused on color preference itself on computer interface of Chinese people systemically.

In this study, we investigated the color preference of User Interface of Chinese youth. We intended to use a better way to study this issue. First, 7 10 colors would be chose from the basic colors pool, using as materials of background color study. Second, background color preference was explored in details using forced choice and subjective evaluation. Third, combining foreground color, we investigated color 
combination preference. Finally, visual performance of color combination was also tested.

\section{Experiment 1}

\subsection{Purpose}

This experiment was designed to choose materials for experiment 2. Participants were asked to choose 10 favorite colors as background color of user interface.

\subsection{Method}

Participants. 20 students from Beijing Normal University ( $\mathrm{N}=10$ male, N=10 female), aged 20 to 23. All participants had normal color vision and normal or correct to normal visual acuity.

Materials. 48 basic colors from the drawing program of windows 98, each color was showed in $20 \times 13 \mathrm{~mm}$ rectangle, were arranged in a $8 \times 6$ matrix. All of them were presented on a screen. The background color was light gray.

Apparatus. The material was presented on a 17-inch color CRT monitor, $1024 \times 768$ pixels , saturation $100 \%$, brightness $50 \%$, red $55 \%$, green $55 \%$, blue $55 \%$ 。

Procedure. We showed the 48 basic colors on the computer screen in one time, the participants were asked to choose 10 favorite colors from them as the background color of computer user interface and to order these colors by their preference.

\subsection{Data Analyze and Results}

42 colors of 48 basic colors were chose as favorite colors in this part. Each color was scored based on their order. For example, if one person chose blue as first color, it was scored 10; if one person chose blue as second color, it was scored 9; the rest may be deduced by analogy. The final score of each color was the sum of every participant's results. The first 10 colors are blue, dark purple, dark blue, gray, white, gray blue, purple, gray purple, cyan and rose. Their scores are 87, 82, 72, 62, $61,60,55,54,49$ and 39 respectively.

A Chi-square test revealed there were significant difference among these 10 colors, $\chi^{2}=31.254, \mathrm{df}=9, P<.001$. Because the purpose of this study was to explore color preference of colorful user interface, gray and white were exclude in next experiment. Finally, blue, dark purple, dark blue, gray blue, purple, gray purple, cyan and rose color were chosen to be the material of experiment 2 .

\section{Experiment 2}

\subsection{Purpose}

This experiment, applying forced choice and subjective evaluation, was designed to investigate the background color preference based on the results of experiment 1 . 


\subsection{Method}

Participants. 44 students from China Agricultural University and Institute of psychology ( $\mathrm{N}=23$ male, $\mathrm{N}=21$ female), aged 19 to 34 , joined in this experiment. All participants had normal color vision and normal or correct to normal visual acuity.

Materials. 8 colors chose in experiment 1 were used to be the materials.

Procedure. Experiment 2 was composed of two parts: Part one: forced choice. In this part, two colors of 8 colors were presented on a screen each time; each color took up half of the screen. Participants were asked to choose their favorite background color in the two colors. If they chose left color, they should press " 1 " on the number keypad; if they chose right color, they should press "2" on the number keypad. In order to exclude location effect, each color compared with other 7 colors both on left and right. The choice time of each color was recorded. There were four times practices before the formal experiment. Part two: subjective evaluation. Participants were asked to rate each color in enjoyable, comfortable and suitable as background color of a computer interface. 7-point scale were used in this part $(1=$ most enjoyable/comfortable/suitable, $7=$ most non- enjoyable/comfortable/suitable). One color presented randomly on the whole screen each time, three scales were showed on right of the screen. Participants were asked to use mouse to choose corresponding score for each color. After evaluated three aspects, participants can press the "NEXT" button at the bottom of the screen to go to evaluate next color.

\subsection{Results}

Forced Choice. Each participant's choice times were transformed to Z score, showed in table 1. Repeated measures of General Linear Model were used to analyze the difference among different colors, $F(7,301)=10.223, P<0.001$.

Table 1. Descriptive results of forced choice

\begin{tabular}{ccccccccc}
\hline color & blue & purple & cyan & $\begin{array}{c}\text { gray } \\
\text { blue }\end{array}$ & $\begin{array}{c}\text { dark } \\
\text { purple }\end{array}$ & $\begin{array}{c}\text { dark } \\
\text { blue }\end{array}$ & $\begin{array}{c}\text { gray } \\
\text { purple }\end{array}$ & rose \\
\hline Z score & 0.52 & 0.36 & 0.30 & 0.25 & -0.18 & -0.38 & -0.47 & -0.48 \\
Z' & 2.35 & 2.19 & 2.13 & 2.08 & 1.65 & 1.45 & 1.36 & 1.35 \\
SD & 0.49 & 0.38 & 0.90 & 0.59 & 0.67 & 1.13 & 0.97 & 0.95 \\
\hline
\end{tabular}

Pairwise comparisons were used to compare the $\mathrm{Z}$ score of each color. The results showed there was significant differece between blue and last 5colors. It was obvious that blue was the most favorite background color for participants. Purple, cyan and gray blue were also different with last 4 colors. It showed that they were also the favorite color for participants.

Subjective evaluation. The result of subjective evaluation in three aspects was showed in fig.1. 
In the enjoyable aspect, the order of background color preference was blue, gray blue, purple, cyan, rose, dark purple, dark blue and gray purple. The results of repeated measures of General Linear Model showed there was significant difference among 8 colors, $F(7,301)=8.895, P<0.001$. Pairwise comparisons results showed there was significant difference between blue and other 7 colors, blue was the most enjoyable background color.

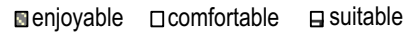

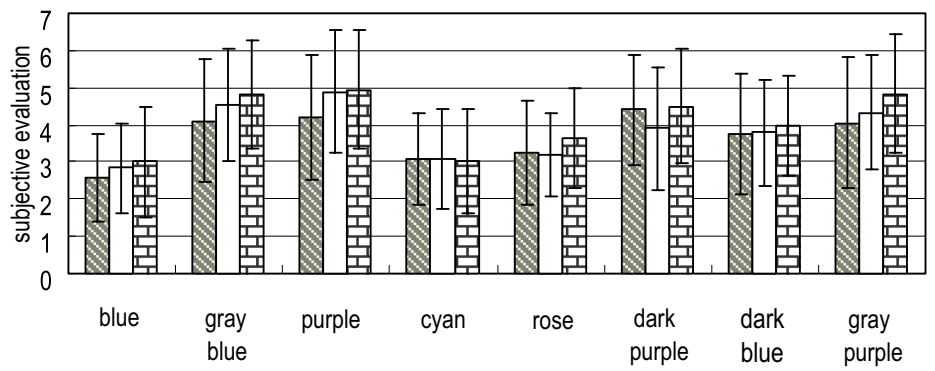

Fig. 1. Subjective evaluation of background colors

In the comfortable aspect, the order of background color preference was blue, gray blue, purple, cyan, gray purple, rose, dark purple and dark blue. The results of repeated measures of General Linear Model showed there was significant difference among 8 colors, $F(7,301)=13.960, P<0.001$. Pairwise comparisons results showed there was significant difference between blue,gray blue,purple and other 5 colors. Blue, gray blue and purple were more comfortable background color.

In the suitable aspect, the order of background color preference was blue, gray blue, purple, cyan, gray purple, dark purple, rose and dark blue. The results of repeated measures of General Linear Model showed there was significant difference among 8 colors, $\mathrm{F}(7,301)=16.038, \mathrm{P}<0.001$. Pairwise comparisons results showed there was significant difference between blue, gray blue, purple, cyan and other 4 colors. Blue, gray blue, purple and cyan were all more suitable as background colors.

The results in three aspects were similar, Spearman correlation coefficient was used to test the correlation of three aspects, there were significant correlation between each two aspects, $\quad r_{\text {enjoyablexsuitable }}=0.932, r_{\text {comfortablexsuitable }}=0.959, \quad r_{\text {enjoyablexcomfortable }}=0.875$. It prompted they could impact each other, so they all need to be considered in the interface design.

Both forced choice and subjective evaluation results showed blue, gray blue, purple and cyan were more favorite to use as background color, so they were used as background color in the next experiment, to explore color combination preference. 


\section{Experiment 3}

\subsection{Purpose}

Subjective evaluation was used to investigate the color combination preference.

\subsection{Method}

Participants. 43 students from China Agricultural University ( $\mathrm{N}=23$ male, N=20 female), aged 19 to 24, joined in this experiment. All participants had normal color vision and normal or correct to normal visual acuity.

Materials and procedure. Blue, gray blue, purple and cyan were used as background colors, other basic colors were used as foreground color. The procedure was similar as subjective evaluation part of experiment 2 . One foreground color was showed in $20 \times 13 \mathrm{~mm}$ rectangle on one background color each time, participants were asked to rate if this combination was suitable as computer user interface on a 7-point scale ( $1=$ most suitable; $7=$ very not suitable). They need to evaluate 184 times at all.

\subsection{Results}

Table 2 showed first 10 color combinations.

Table 2. Descriptive results of first 10 color combinations

\begin{tabular}{ccccccccccc}
\hline $\begin{array}{l}\text { Foreground Blue/ Blue/ } \\
\text { Background } \\
\text { white }\end{array}$ & $\begin{array}{c}\text { Blight } \\
\text { yellow }\end{array}$ & $\begin{array}{c}\text { Blue/ } \\
\text { cyan }\end{array}$ & $\begin{array}{c}\text { Gray } \\
\text { grass } \\
\text { green } \\
\text { white }\end{array}$ & $\begin{array}{c}\text { Blue/ } \\
\text { light } \\
\text { green }\end{array}$ & $\begin{array}{c}\text { Purple/ Purple/ } \\
\text { white } \\
\text { light } \\
\text { yellow }\end{array}$ & $\begin{array}{c}\text { Blue/ Blue/ } \\
\text { green yellow }\end{array}$ \\
\hline Mean & 2.95 & 3.26 & 3.28 & 3.37 & 3.37 & 3.4 & 3.47 & 3.51 & 3.53 & 3.53 \\
SD & 1.59 & 1.57 & 1.71 & 1.9 & 1.96 & 1.68 & 1.86 & 1.67 & 1.91 & 1.72 \\
\hline
\end{tabular}

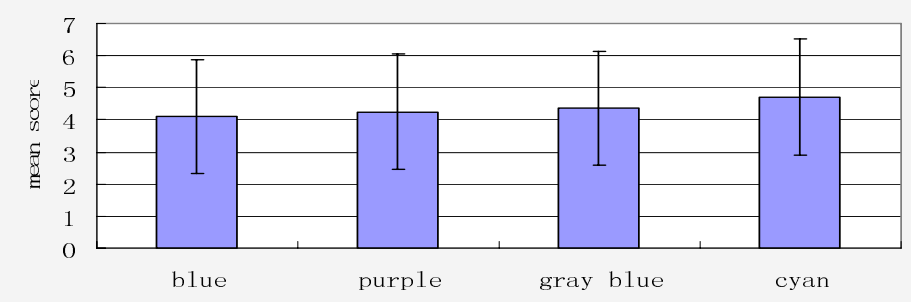

Fig. 2. Subjective evaluation among different background color

It was obvious that blue was the favorite background color, purple was also popular. Cyan was not chosen into first 10 combinations. Repeated measures of General Linear Model were used to analyze the difference among four background 
colors, descriptive results showed in fig. $2, F(3,126)=6.971, P<.01$, the combinations in which blue was used as the background color were the favorite combinations for the participants. It prompted that the color combination preference was impacted by background color.

For the foreground colors, white series, yellow series and green series were all popular foreground colors when the background color was blue, purple or gray-blue. But these results were just observed from descriptive results, more research was needed in the future.

\section{Discussions}

Results of background color preferences experiment showed, blue, purple, cyan and gray blue were chosen as participants' favorite background colors for computer user interface. Although the order of these 4 colors in two methods was not all the same, there were significant difference between them and other 4 colors. Blue was the most popular background color, results of forced choice and subjective evaluation were both proved this. This may be related with the characteristic of blue, some research prompted blue can inspire positive emotion, e.g. comfort, stillness et al. (Wu \& Wang $^{[8]}$, 1986; Huang et al. $\left.{ }^{[9]}, 1991\right)$. Another reason was participants' habit, the most popular computer user interface, which was used in China widely, was using blue as its main hue (e.g. Windows 98, 2000, XP). Three aspects of subjective evaluation revealed suitable background color was high related with its comfortable and enjoyable degree. If a color was suitable as background color of interface, participants would consider if it was comfortable and if he/she like it. There were some prompts for interface design. But Zhu and Cao (1994) found there was some discord in definition and comfort. How to apply these factors properly was a meaningful question.

Based on the results of color combination preference, foreground color preferences were influenced by background color, blue was still the most favorite background color. This was coincident with common computer interface (Microsoft Windowed series). The foreground color preference was influenced by the background color. White, yellow series and green series were preferred to be favorite foreground colors. White with blue, white with gray blue and white with purple were the best combination in these three background colors. These rules needed to be considered in interface design.

\section{Conclusions}

In a word, our works indicated that blue was the best background for young computer users, and purple, gray-blue and cyan were also good choices. Color combination preference was influenced by background color. White series, yellow series and green series were all popular foreground colors for the background color of blue, purple or gray-blue. 


\section{References}

1. Zeng, H.W.: Color preference of Chinese students and its development [J]. Psychology science communication (in Chinese) 9(1), 16-22 (1986)

2. Wang, Y.R.: An experimental study of color preference of Chinese University students [J]. Psychology science (in Chinese) 20(6), 559-560 (1997)

3. Li, W.F.: Color preference of Chinese townsmen: the relationship between color preference and some factors [J]. Psychology science communication (in Chinese) 13(5), 13-19 (1990)

4. Ellis, L., Fricek, C.: Color preferences according to gender and sexual orientation [J]. Personality individual differences 31(8), 1375-1379 (2001)

5. Zhu, Z.X., Cao, L.R.: The effect of object-background color combination to visual efficiency of color CRT [J]. Transactions of Psychology (in Chinese) 26(2), 128-135 (1994)

6. Cao, L.R., Zhu, Z.X.: The effect of contrast to visual efficiency of color CRT [J]. Ergonomics (in Chinese) 1(1), 32-36 (1995)

7. Kong, Y., Ge, L.Z., Wang, Y.J.: A comparison study of salient efficiency of 4 colors on black and white background [J]. Ergonomics (in Chinese) 5(4), 12-14 (1999)

8. Wu, X.Z., Wang, X.Y.: An investigation of "color-emotion" in university students [J]. Psychology science communication (in Chinese) 9(1), 38-40 (1986)

9. Huang, X.T., Huang, W., Li, X.R.: A color-emotion study of Chinese people [J]. Psychology science (in Chinese) 14(6), 1-7 (1991) 